\title{
Further Evaluation of the Mother-to-Child Transmission Potential of SARS-CoV-2 Infection During Pregnancy: A Retrospective Study
}

\author{
Xuechen Yu \\ Zhongnan Hospital of Wuhan University \\ Huijun Chen \\ Zhongnan Hospital of Wuhan University \\ Fan Luo \\ School of Basic Medical Sciences, Wuhan University \\ Juanjuan Guo \\ Zhongnan Hospital of Wuhan University \\ Yuan Qiao \\ Zhongnan Hospital of Wuhan University \\ Wei Zhang \\ Northwestern University Feinberg School of Medicine \\ Jiafu Li \\ Zhongnan Hospital of Wuhan University \\ Dongchi Zhao \\ Zhongnan Hospital of Wuhan University \\ Dan Xu \\ Zhongnan Hospital of Wuhan University \\ Qing Gong \\ Zhongnan Hospital of Wuhan University \\ Lingyun Yang \\ Zhongnan Hospital of Wuhan University \\ Pu Yang \\ Zhongnan Hospital of Wuhan University \\ Yuming Cao \\ Zhongnan Hospital of Wuhan University \\ Xue Wen \\ Zhongnan Hospital of Wuhan University \\ Wei Hou \\ School of Basic Medical Sciences, Wuhan University \\ Huixia Yang \\ Peking University First Hospital \\ Yuanzhen Zhang ( $\square$ zhangyuanzhen@vip.sina.com) \\ Zhongnan Hospital of Wuhan University https://orcid.org/0000-0003-4832-7902
}

Research article

Keywords: Corona Virus Disease 2019 (COVID-19), severe acute respiratory syndrome coronavirus 2 (SARS-CoV-2), pregnancy, mother-tochild transmission

Posted Date: October 6th, 2020

DOI: https://doi.org/10.21203/rs.3.rs-77490/v1

License: (c) (1) This work is licensed under a Creative Commons Attribution 4.0 International License. Read Full License 


\section{Abstract}

Background. This study aimed to comprehensively evaluate the clinical characteristics of COVID-19 in perinatal period, and systematically assess the mother-to-child transmission potential of SARS-CoV-2

Methods. We retrospectively analyzed the data of 23 pregnant patients in late pregnancy. Clinical specimens, including maternal and neonatal throat swabs, vaginal secretions, placenta tissues, and breast milk, were collected for the nucleic acid test of the virus. Pregnancy outcomes and neonatal results were also analyzed.

Results. Overall, 10 patients (43.5\%) had no symptoms and were found by routine chest CT. Complications appeared after COVID-19 onset included PROM (17.4\%) and fetal distress (4.3\%). Typical signs of viral pneumonia were recorded in chest CT of all patients. No patients developed severe pneumonia or died of COVID-19. All of 25 neonates were born alive. No severe asphyxia or neonatal death was observed. Although three neonates were tested transiently suspected positive for SARS-CoV-2 after being transferred to neonatology department, no newborns developed COVID-19. Out of various clinical specimens tested, only a rectal swab sample from one pregnant patient was tested positive for SARS-CoV-2, while all the other specimens including first sample of newborn throat swabs were negative. Pathological examination found no obvious chorioamnionitis or clear virus inclusion body in placenta, and ACE2 (angiotension-converting enzyme 2) was expressed at a moderate level.

Conclusions. As in the general population of COVID-19, asymptomatic patients were present in pregnant women. There is no confirmatory evidence for mother-to-child transmission in COVID-19 patients with late pregnancy.

\section{Background}

The Corona Virus Disease 2019『COVID-19『 cased by the Severe Acute Respiratory Syndrome Coronavirus 2 (SRAS-CoV-2) has become a global pandemic. There have been over 3 million confirmed cases ${ }^{1}$ from all populations including women with pregnancy. As maternal diseases could cause adverse effects on the fetus or newborn, whether SARS-CoV-2 can be transmitted from mother to child has become one of the most concerned issues in the current pandemic. Our previous report has described the clinical characteristics of 9 pregnant women with laboratory-confirmed COVID-19 and found no direct evidence for the existence of SARS-CoV-2 in amniotic fluid, cord blood, and newborn throat swab samples ${ }^{2}$. However, the definition of mother-to-child transmission may also refer to the intrapartum transmission, which can be resulted from the contact of newborn with virus-contaminated maternal birth canal during vaginal delivery, as well as the postpartum transmission that can be caused by breastfeeding and intimate contact with infected mothers. Prior studies including ours lacked data from vaginal secretions and placenta specimens. Additionally, two recent reports on ACE2 (angiotension-converting enzyme 2) receptor expression in placental samples from COVID-19 patients drew inconsistent conclusions ${ }^{3,4}$. Therefore, more comprehensive and systematic studies would be necessary to re-evaluate this important issue and determine the possibility of mother-to-child transmission of SARS-CoV-2. In the current study, we further analyzed the clinical characteristics of SARS-CoV-2 patients with pregnancy using an expanded cohort of 23 cases, all of whom were in their third trimester. Notably, we systematically collected various types of clinical specimens potentially relevant to mother-to-child transmission, including vaginal secretions, rectal swabs, and breast milk, in addition to other specimens essential for estimating vertical transmission, such as amniotic fluid, cord blood, placenta, and newborn throat swab samples that were collected immediately after birth. The presence of SARS-CoV-2 in these clinical specimens was determined using the nucleic acid test. Findings from this study updated our understanding of potential vertical transmission in pregnancy and provided clinical guidance to the perinatal treatment of the SARS-CoV-2 infected pregnant women, especially for the delivery protection and the neonatal care.

\section{Methods}

\section{Study design and participants}

This is a single center study from Zhongnan Hospital of Wuhan University, which was a designated medical institution for pregnant patients with COVID-19. The clinical data of 23 patients with laboratory confirmed SARS-CoV-2 infection were analyzed retrospectively. The patients were hospitalized in the Department of Obstetrics from February 6 to March 5, 2020 and were followed up until March 16, 2020(a median follow-up of 31 days). The diagnosis of COVID-19 was based on the "Diagnosis and Treatment Program of Novel Coronavirus Pneumonia New Coronavirus" (Trial Version 7) published by the National Health Commission of China ${ }^{5}$. Suspected cases with positive SARS-CoV-2 nucleic acid or SARS-CoV-2-lgM/lgG were defined as confirmed cases. The study was approved by the Medical Ethics Committee of Zhongnan Hospital of Wuhan University (approval No. 2020031). Written consent was obtained from each patient.

The data was obtained from the electronic medical record system and was verified through direct communication with the patients and their attending doctors. Two trained researchers (XY and JG) verified the data accuracy using a designed data collection form independently. 
Clinical specimens were processed according to an updated protocol from our prior study ${ }^{2}$. Briefly, maternal throat swab samples were collected immediately after admission. Rectal and vaginal secretion samples of the mothers were gathered before delivery. Amniotic fluid samples were abstained by direct syringe aspiration before rupture of fetal membrane during cesarean section, or at artificial rupture during vaginal delivery. Cord blood samples were extracted after clamping the umbilical cord. The throat swabs and rectal samples of newborns were collected in the operating room or delivery room immediately after birth. Two placenta tissue samples were obtained from each patient. One was fixed with $10 \%$ neutral formaldehyde for 24 hours and then embedded in paraffin. The other was frozen in liquid nitrogen and stored at $-80^{\circ} \mathrm{C}$. Additionally, breast milk samples from the mothers were collected after their lactation by extrusion.

\section{RT-PCR for SARS-CoV-2}

All samples were processed in accordance with the WHO guidelines ${ }^{6}$. Laboratory test of SARS-CoV-2 in maternal and neonatal throat swabs were conducted by the Department of Clinical laboratory, Zhongnan Hospital using the kit recommended by China Center for Disease Control and Prevention (BioGerm, Shanghai, China). Positive results were determined when the two tested targets of SARS-CoV-2 (Nucleoprotein gene, ORF1ab) were detected at the same time. Suspected results were those when only one target was positive, or there was amplification reaction when the test result was under the detection limit. Serological detection was also completed by the Department of Clinical Laboratory using colloidal gold Kit (Neo Nostics Bioengineering Co., Suzhou, China). Other clinical specimens were detected by the State Key Laboratory of Virology/Institute of Medical Virology, School of Basic Medical Sciences, Wuhan University through nested RT-PCR with designed primers. The first neonatal throat swab samples, which were obtained in the operating room or delivery room, were also tested for the viral nucleic acids by nested RT-PCR. The details of experiments were as follows: Trizol LS (Invitrogen, Carlsbad, CA, USA) was used to extract the total RNA from the samples, and then one-step RT-PCR Kit (Takara, Dalian, China) was applied for reverse transcription. Primers were designed based on the reported SARS-CoV-2 sequence (Wuhan-Hu-1, MN908947), in order to amplify partial s-segment sequence (nt21730-22458). The specific primers used here were 5 '- CTCAGGACTTGTTCTTACCTT -3', 5 '- CAAGTGCACAGTCTACAGC -3 '.

\section{Immunohistochemistry for ACE2}

The immunohistochemistry of ACE2 was performed as previously described ${ }^{7}$. Briefly, 4-mm thick sections of formalin-fixed/paraffinembedded specimens were deparaffinized and rehydrated according to standard protocols. Sections were blocked with rabbit serum and incubated with antibodies against ACE2 (OriGene Technologies, Rockville, Maryland, USA). Antigens were visualized by the streptavidinbiotin-peroxidase complex method. Immunostaining was evaluated by two pathologists without knowledge of a patient's clinical information. Extent of immunostaining was graded on the basis of the percentage of cells displaying staining. The number of staining cells less than $10 \%$ was considered as "low level", while 10\%-50\% and >50\% were considered as "moderate" and "high level", respectively.

\section{Statistical analysis}

The SPSS 20.0 software was used for statistical analysis. The classification variables were expressed as percentage (\%) and compared by the $\chi^{2}$ test or Fisher exact test. If a continuous variable was in normal distribution, it was expressed as mean value and standard deviation (SD) and compared with the Student's t-test. If a continuous variable was not normally distributed, it was represented as median and interquartile ranges (IQR) and compared using the Mann-Whitney $U$ test.

\section{Results}

\section{Clinical characteristics of pregnant women with COVID-19}

The median age of enrolled patients was 30 years (IQR, 30-32; range, 27-38 years) (Table 1). All of the patients resided in Wuhan. However, 13 of them (56.5\%) did not recall a history of exposure except their experience of visiting hospitals or local supermarkets. One case (4.3\%) showed familial aggregation. They were all in late pregnancy at admission, and their median gestational weeks (GWS) was $38^{+5}$ weeks (IQR, $37^{+6}-39^{+4}$; range, $31^{+6}-41^{+2}$ weeks). Among the 23 patients, 21 had full-term pregnancy. Of the two patients who did not reach full-term pregnancy, one case was complicated with sever preeclampsia at $32 \mathrm{GWS}$. Another case with twin pregnancy hospitalized in emergency due to placental abruption at $31^{+6} \mathrm{GWS}$. In addition, there were $18(78.3 \%)$ cases who had pregnancy complications (Table 1$)$, the most common of which were pregnancy-induced hypertension (5 cases, $21.7 \%$ ) and gestational diabetes (2 cases, $8.7 \%$ ). Other complications, such as PROM (4 cases, $17.4 \%$ ) and fetal distress (1 case, $4.3 \%$ ), which appeared after COVID-19 onset were also observed. In addition, 4 cases were hepatitis virus carriers (4 cases, $17.4 \%$ ).

Only 13 cases (56.5\%) had typical symptoms of SARS-CoV-2 infection, of which 10 (43.5\%) had fever and 6 (26.1\%) presented cough. Ten patients (43.5\%) did not manifest any symptoms before admission. Their signs of infection were detected with routine CT, and then confirmed by the nucleic acid test (Table 1). As shown in Table 1, there were 14 cases (60.9\%) with normal leukocytes, 15 cases (65.2\%) with 
increased neutrophils, and 5 cases (21.7\%) with decreased lymphocytes. A small proportion of patients (22.7\%) had elevated C-reactive protein (CRP). Other abnormalities of laboratory examination included slight increase of alanine aminotransferase (ALT, 8.7\%), aspartate aminotransferase (AST, 13.0\%), creatine kinase isoenzyme (2 case, 9.1\%), and lactate dehydrogenase (LDH, 4 cases, $18.2 \%)$. Interleukin 6 (IL6) was detected in 21 patients, and 9 of them (42.9\%) showed IL-6 levels above the normal range. In addition, two (15.4\%) of the 13 patients who received influenza virus test had positive results. All patients underwent chest CT scan and showed typical signs of viral pneumonia.

All cases were terminated pregnancy within 24 hours after admission. One case experienced vaginal delivery, while the other 22 cases underwent cesarean section in negative pressure operating room. Among them, two patients had entered the active phase and been in trial labor for 5 hours and 8 hours, respectively. They finally were subjected to cesarean sections because they were not able to tolerate wearing masks any longer. Common antibiotics (cephalosporin) were routinely given to operative patients for two days. Then they were transferred to the Department of Infectious Diseases or other designated hospitals for further treatment several days after delivery. Hormones were not used during the period of hospitalization in the Department of Obstetrics. One case developed severe postpartum thrombocytopenia 12 hours after operation with unknown causes and was relieved by intravenous administration of two doses of immunoglobulin (total dose $40 \mathrm{~g}$ ). As of March 16, 2020 (a median follow-up of 31 days), no patients developed severe pneumonia, needed intensive care, or died of COVID-19.

\section{Neonatal outcomes}

A total of $25(100 \%)$ neonates, including two pairs of twins, were born alive. Twenty-four newborns were transferred to the negative pressure ward of the Department Neonatology for isolation and systematical evaluation. Only one case refused transfer, and that newborn was taken home by his family. There was no severe asphyxia or neonatal death in all newborns. Even three preterm newborns had a 1-minute Apgar score $\geq 7$ and a 5-minute Apgar score $\geq 8$. One case was born prematurely at 32 GWS due to maternal severe preeclampsia. As of March 16, 2020, she still stayed in the Department of Neonatology in stable condition. Another pair of preterm twins was given birth through emergency cesarean section because of placental abruption and was discharged without complications 29 days later.

The newborns received first throat swab sampling in the operating room and the results were all negative for SARS-CoV-2. Then, the 24 cases, who were transferred to the Department of Neonatology, experienced more throat swab tests during observation. Two newborns showed suspected results in the second or third SARS-CoV-2 test, which was performed several hours after birth (3 and 19 hours, respectively), but then showed negative results for another three times on different dates. The results of the third newborn, who was one of the premature twins, showed a similar pattern. Of his four times of SARS-CoV-2 tests, only the one performed 3 hours after birth was suspected positive. In contrast, his twin sister had negative results in all four tests. All these suspected positive results could not be repeated thereafter. Importantly, all the newborns were examined by chest X-ray, and no signs of pulmonary infection were found.

\section{Mother-to-child transmission potential of SARS-CoV-2}

The existence of SARS-CoV-2 in maternal vaginal secretions and rectal swabs was tested by RT-PCR before pregnancy termination. Out of all samples tests, only a rectal swab sample from one pregnant patient showed a positive result, and all the other samples were negative for the viral nucleic acids. Clinical samples, including amniotic fluid, cord blood, placenta and newborn throat swabs were collected and processed immediately after birth. Similar to our prior report ${ }^{2}$, no evidence for SARS-CoV-2's nucleic acids was found in all of these samples from mothers and newborns. In addition, no obvious chorioamnionitis or clear virus inclusion body was found in the histopathological section of placenta (Fig. 1). The results of immunohistochemistry showed that ACE2 protein was expressed in placenta with low or moderate level (Fig. 2). Finally, breast milk was obtained postpartum, and no SARS-CoV-2 was detected by RT-PCR assay.

\section{Discussion}

So far, there have been no studies with a large sample size to convincingly summarize the clinical features of COVID-19 in pregnancy. Based on the data of 9 cases, we demonstrated that pregnant patients with COVID-19 had the comparable symptoms as non-pregnant adults ${ }^{2}$. In the current report, we described more comprehensively the clinical characteristics of COVID-19 patients with pregnancy with an expanded patient cohort of 23 cases in their third trimester. Fever and cough were still the most common symptoms, consistent with our previous findings. However, nearly half of the patients manifested no typical symptoms of SARS-CoV-2 infection. Instead, they just showed abnormal CT images (43.5\%), which was the only clue for us to discover these asymptomatic infections. The results of laboratory examination were also atypical. It was showed that $39.1 \%$ of the patients had increased leukocytes, $65.2 \%$ had increased neutrophils, and $21.7 \%$ had decreased lymphocytes, showing some differences from what was reported by Chen et al ${ }^{8}$. Although the nucleic acid test is still used as the "gold standard" for the diagnosis of COVID-19, it is not unusual that patient compliance and the way of sampling as well as storage of throat swabs may lead to false negatives. Furthermore, there is a so-called "window period" for the detection of virus serology, which can be affected by the differences of individual immune response and other factors. In contrast, chest CT showed a better sensitivity than nucleic

Page 4/12 
acid test in the early detection of SARS-CoV-2 infection ${ }^{9}$. Thus, we suggested that CT should be included in routine screening for pregnant women requiring hospitalization, as the safety of chest CT in pregnancy has been verified ${ }^{10}$. Moreover, in order to avoid the adverse consequences of patients with asymptomatic infection, it is necessary to adopt the highest level of protective measures for emergency patients who did not complete the screening in time.

Mother-to-child transmission, which is the most serious adverse effect for pregnant women with pathogen infection, can take place through several channels. With regard to vertical transmission, various viruses behave differently. Before the discovery of SARS-CoV-2, six coronaviruses were known to infect human beings, four of which, including HCoV-229E, HCoV-OC43, HCoV-NL63, as well as HCoV-HKU1, usually showed common cold symptoms and could be transmitted vertically ${ }^{11}$. On the contrary, there is no evidence to support the vertical transmission of SARS-CoV or MERS-CoV ${ }^{12-14}$. Our previous study demonstrated no evidence for the existence of SARS-CoV-2 in amniotic fluid, cord blood and newborn throat swabs by RT-PCR ${ }^{2}$. Similar results have been reported in subsequent studies independently ${ }^{15}$. However, limited samples were reported so far, and the data with placenta was still inadequate and controversial. Here, in addition to the negative results of SARS-CoV-2 in cord blood, amniotic fluid and newborn throat swab samples from 23 cases, we also demonstrated that no viral nucleic acids could be detected in placenta tissue, which provided stronger evidence for the absence of vertical transmission. The damage of placenta structure is also the reason for adverse pregnancy outcomes. In the current study, no virus inclusion bodies were found in placenta, and no obvious destruction of placenta structure were observed.

In the context of the WHO's efforts to promote vaginal delivery ${ }^{16}$, it is of great significance to clarify whether SARS-CoV-2 can transmit to the newborn through vagina during delivery. However, it is not appropriate to expose the newborn and medical staff in an environment that may cause infection for a long time, in the case of without knowing the risk of intrapartum transmission. Because COVID-19 is a severe respiratory infectious disease, and trial labor is a time-consuming and energy consuming dynamic process with variable outcomes, pregnant women may open their masks to breathe because of oxygen consumption, or cry out for pain, both of which can result in generation of viral aerosols. Two cases in this study chose caesarean just because they were not able to tolerate wearing masks anymore. To give a preliminary risk assessment about intrapartum transmission of SARS-CoV-2, we detected the samples of vaginal secretions for SARS-CoV-2and obtained negative results from all cases. This provided us more supporting evidence that neonates would not be infected during delivery due to the contacts with maternal birth canal. What's more, the newborn who was born through vaginal delivery had no signs of any SARS-CoV-2 infection. Thus, vaginal delivery should be taken into account, at least for those patients who have entered the stage of labor and can end the labor in a short time, because the risk of pathogens entering the fetus due to uterine contraction no longer can be eliminated. The prerequisite of vaginal trial is to strictly protect the area around the birth canal. After all, there have been studies in which the existence of virus from feces was detected $^{17,18}$. Our study also showed a case with SARS-CoV-2 positive in her perianal secretion sample. In the process of delivery, protecting the birth canal from contamination, avoiding invasive operation, clamping the umbilical cord quickly and separating the newborn from maternal surrounding environment as soon as possible, may help to reduce the risk of newborn's exposure to virus and the possibility of infection.

Since the lack of evidence of vertical transmission and intrapartum transmission, we should put our focus of neonatal protection on postpartum transmission. However, during the process of early neonatal care after umbilical cord clamping, there was no protective measure for newborn. Besides that, because the newborns of infected mother were defined as suspected cases, they should be transferred through the patient channel. Although the total transit time was only a few hours, the possibility of exposure to the virus could not be completely ruled out. Recently, there have been reports about cases of neonatal infection, but they did not clarify the transport process after birth and the time of samples collection, so it is not appropriate to infer that these newborns were infected through vertical transmission. It has been recommended that, in the study of mother-to-child transmission, samples of placenta, amniotic fluid, cord blood and nasopharynx swabs should be taken for detection immediately after delivery in sterile environment. Only if we get the necessary samples correctly and carry out standard detection, can the scientific evidence be obtained. In other word, the positive nasopharynx swab results of newborns with transport experience do not indicate that the virus was obtained from their mothers. In this study, SARS-CoV-2 tests in several newborns were negative at birth, but positive after being transported to neonatal department for several hours. However, this positive result could not be repeated in the next few days. This may because these newborns were exposed to a small amount of virus during transport, or the results were false positive. Special neonatal transport system should be designed and used for newborns of COVID-19 patients.

The transmissibility of SARS-CoV-2 through breast milk is another issue of widespread concern of health practitioners and policy makers. If breastfeeding is wrongly discouraged, years of public health efforts to promote breastfeeding could be lost. Conversely, if breastfeeding is wrongly encouraged, many infants could be put at risk. For instance, Ebola virus has been found in the breast milk of mothers in acute and convalescent phases of Ebola Virus Disease (EVD) as well as in asymptomatic mothers ${ }^{19}$. Thus, breastfeeding should not be suggested in women with EVD. Consistent with our previous results, SARS-CoV-2 was not detected in the breast milk of infected women. However, other factors such as sucking of the newborn should also be taken into account for breastfeeding. Maternal saliva, respiratory droplets and even 
aerosols may cause neonatal infection. Taken together, we would like to advise mothers with SARS-CoV-2 infection to squeeze milk into sterile containers under protection, and the infants should be fed by healthy family members. Direct breastfeeding in COVID-19 patients should be opposed.

We acknowledge that the significance of this study is limited by several factors. First of all, none of the enrolled patients had been infected by SARS-CoV-2 for a long time and developed severe pneumonia before delivery. Thus, it could not rule out that the placental barrier of severe ill patients with viremia might be destroyed due to inflammation as well as necrosis, and the virus may enter uterine cavity to cause fetal infection. Secondly, the outcomes of infants from patients who were infected in the first or second trimester of pregnancy remain unclear. Thirdly, the risk of intrapartum infection should be assessed with more cases of vaginal delivery. Finally, the long-term prognosis of newborns of COVID-19 patients is not known and a systematic follow-up is needed.

\section{Conclusions}

In conclusion, the clinical characteristics of pregnant women with SARS-CoV-2 infection were atypical. More importantly, there were fair number of asymptomatic infections. Thus, a routine screening, consisting of chest CT, nucleic acid and serological test, was recommended to be carried out in women hospitalized for labor to prevent nosocomial infection. Neither evidence for vertical transmission nor for intrapartum transmission were found in COVID-19 patients with late pregnancy. Measures, such as protecting the birth canal from contamination, are helpful to avoid intrapartum transmission. Meanwhile, strict protection in the transport process is the most important aspect in preventing postpartum transmission. In addition, the absence of SARS-CoV-2 in breast milk provide a support for indirect feeding. The highlights of this study were systematically evaluated the risks of mother-to-child transmission of SARS-CoV-2 with the largest sample size so far. Considering the current epidemic of COVID-19 in the world, although the significance of our work is limited by sample size and prospective method, we think that our findings provide valuable basis for making perinatal treatment guidelines in COVID-19 patients.

\section{Abbreviations}

COVID-19: Corona Virus Disease 2019; SRAS-CoV-2: Severe Acute Respiratory Syndrome Coronavirus 2; ACE2: Angiotension-converting enzyme 2; RT-PCR: Reverse transcription polymerase chain reaction; CT: Computerized tomography; CRP: C-reactive protein; ALT: Alanine aminotransferase; AST: Aspartate aminotransferase; LDH: Lactate dehydrogenase; WHO: World Health Organization; EVD: Ebola Virus Disease

\section{Declarations}

\section{Acknowledgments}

This study was supported by Hubei Provincial Science and Technology Department Novel Coronavirus Pneumonia Emergency Science and Technology Project (Grant No. 2020FCA011), Wuhan Novel Coronavirus Pneumonia Emergency Science and Technology Tackling Key Project (Grant No. 2020020201010011)

\section{Authors' contributions}

YZ, HY and WH had made substantial contributions to concepts and design. XY and HC were in charge of the manuscript draft and revising it based on reviewers' comments. $X Y, J G$ and $Y Q$ made substantial contributions to data acquisition. $X Y$ was in charge of data analysis and interpretation. JG, YC and XW take the responsibility of getting written consents of the patients, applying ethical approval, collecting samples and confirming data accuracy. JL, YQ, DX, QG and LY were the obstetricians of the patients, DZ and PY were the pediatricians of the newborns, they were responsible for data collection and confirmation. WH and FL were responsible for the laboratory task, including sample processing and detection. WZ made substantial revision of the manuscript.

\section{Funding}

This study was supported by Hubei Provincial Science and Technology Department Novel Coronavirus Pneumonia Emergency Science and Technology Project (Grant No. 2020FCA011), Wuhan Novel Coronavirus Pneumonia Emergency Science and Technology Tackling Key Project (Grant No. 2020020201010011). The funding source was not involved in this study.

\section{Ethics approval and consent to participate}

The study was approved by the Medical Ethics Committee of Zhongnan Hospital of Wuhan University (approval No. 2020031). Written consent was obtained from each patient. 
Data can be provided after the article is published. Once the data can be made public, the research team will provide an email address for communication. The corresponding author has the right to decide whether to share the data or not based on the research objectives and plan provided. With the permission of the corresponding author, we can provide participant data without names and identifiers but not study protocol, statistical analysis plan or informed consent form.

\section{Consent for publication}

Not applicable.

\section{Competing interests}

The authors declare that there are no conflicts of interest in this manuscript.

\section{Author details}

${ }^{1}$ Department of Gynaecology and Obstetrics, Zhongnan Hospital of Wuhan University, Clinical Medicine Research Center of Prenatal Diagnosis and Birth Health in Hubei Province, Wuhan, 430071, China

${ }^{2}$ State Key Laboratory of Virology/Institute of Medical Virology, School of Basic Medical Sciences, Wuhan University, Wuhan, 430071, China

${ }^{3}$ Department of Preventive Medicine, Northwestern University Feinberg School of Medicine, Chicago, USA

${ }^{4}$ Department of Pediatrics, Zhongnan Hospital of Wuhan University, Wuhan, 430071, China

${ }^{5}$ Department of Obstetrics and Gynecology of Peking University First Hospital, Beijing, 100034, China

\section{References}

1. Coronavirus disease 2019 (COVID-19) Situation Report-89. Available from: https://www.who.int/docs/defaultsource/coronaviruse/situation-reports/20200418-sitrep-89-covid-19.pdf?sfvrsn=3643dd38_2 (accessed Apr 19, 2020).

2. Chen $\mathrm{H}$, Guo J, Wang C, et al. Clinical characteristics and intrauterine vertical transmission potential of COVID-19 infection in nine pregnant women: a retrospective review of medical records [published correction appears in Lancet. 2020 Mar 28;395(10229):1038] [published correction appears in Lancet. 2020 Mar 28;395(10229):1038]. Lancet. 2020;395(10226):809-815. doi:10.1016/S01406736(20)30360-3.

3. Li M, Chen L, Zhang J, Xiong C, Li X. The SARS-CoV-2 receptor ACE2 expression of maternal-fetal interface and fetal organs by single-cell transcriptome study. PLoS One. 2020;15(4):e0230295. Published 2020 Apr 16. doi:10.1371/journal.pone.0230295.

4. Zheng Q L, Duan T, Jin L P. Single-cell RNA expression profiling of ACE2 and AXL in the human maternal-Fetal interface. Reprod Dev Med, 2020; published online Mar 13.

5. Chinese Center for Disease Control and Prevention. Diagnosis and Treatment Program of Novel Coronavirus Pneumonia New Coronavirus (Trial version 7) Feb 18, 2020. Available from: http://www.nhc.gov.cn/yzygj/s7653p/202003/46c9294a7dfe4cef80dc7f5912eb1989/files/ce3e6945832a438eaae415350a8ce964.pdf. (accessed Mar 22, 2020).

6. World Health Organization (WHO). Laboratory testing for 2019 novel coronavirus (2019-nCoV) in suspected human cases. Interim guidance. Geneva: WHO; 17 Jan 2020.https://www.who.int/health-topics/coronavirus/laboratory-diagnostics-for-novel-coronavirus

7. Valdés G, Neves LA, Anton L, et al. Distribution of angiotensin-(1-7) and ACE2 in human placentas of normal and pathological pregnancies. Placenta. 2006;27(2-3):200-207. doi:10.1016/j.placenta.2005.02.015.

8. Alserehi H, Wali G, Alshukairi A, Alraddadi B. Impact of Middle East Respiratory Syndrome coronavirus (MERS-CoV) on pregnancy and perinatal outcome. BMC Infect Dis. 2016;16:105. Published 2016 Mar 2. doi:10.1186/s12879-016-1437-y.

9. Ai T, Yang Z, Hou H, et al. Correlation of Chest CT and RT-PCR Testing in Coronavirus Disease 2019 (COVID-19) in China: A Report of 1014 Cases [published online ahead of print, 2020 Feb 26]. Radiology. 2020;200642. doi:10.1148/radiol.2020200642.

10. Tomà $P$, Bartoloni $A$, Salerno $S$, et al. Protecting sensitive patient groups from imaging using ionizing radiation: effects during pregnancy, in fetal life and childhood. Radiol Med. 2019;124(8):736-744. doi:10.1007/s11547-019-01034-8. 
11. Gagneur A, Dirson E, Audebert S, et al. Materno-fetal transmission of human coronaviruses: a prospective pilot study. Eur J Clin Microbiol Infect Dis. 2008;27(9):863-866. doi:10.1007/s10096-008-0505-7.

12. Ng PC, Leung CW, Chiu WK, Wong SF, Hon EK. SARS in newborns and children. Biol Neonate. 2004;85(4):293-298. doi:10.1159/000078174.

13. Chen N, Zhou M, Dong X, et al. Epidemiological and clinical characteristics of 99 cases of 2019 novel coronavirus pneumonia in Wuhan, China: a descriptive study. Lancet. 2020;395(10223):507-513. doi:10.1016/S0140-6736(20)30211-7.

14. Stockman LJ, Lowther SA, Coy K, Saw J, Parashar UD. SARS during pregnancy, United States. Emerg Infect Dis. 2004;10(9):1689-1690. doi:10.3201/eid1009.040244.

15. Zhu H, Wang L, Fang C, et al. Clinical analysis of 10 neonates born to mothers with 2019-nCoV pneumonia. Transl Pediatr. 2020;9(1):51-60. doi:10.21037/tp.2020.02.06.

16. WHO Recommendations on Health Promotion Interventions for Maternal and Newborn Health. Geneva: World Health Organization; 2015.

17. Holshue ML, DeBolt C, Lindquist S, et al. First Case of 2019 Novel Coronavirus in the United States. N Engl J Med. 2020;382(10):929936. doi:10.1056/NEJMoa2001191.

18. Wu Y, Guo C, Tang L, et al. Prolonged presence of SARS-CoV-2 viral RNA in faecal samples. Lancet Gastroenterol Hepatol. 2020;5(5):434-435. doi:10.1016/S2468-1253(20)30083-2.

19. Vetter P, Fischer WA 2nd, Schibler M, Jacobs M, Bausch DG, Kaiser L. Ebola Virus Shedding and Transmission: Review of Current Evidence. J Infect Dis. 2016;214(suppl 3):S177-S184. doi:10.1093/infdis/jiw254.

\section{Tables}

Table 1: Baseline Characteristics $\square$ Clinical Characteristics and Treatment of Patients Infected With SARS-CoV-2 


\begin{tabular}{|c|c|}
\hline & Total $(\mathrm{N}=23)$ \\
\hline \multicolumn{2}{|l|}{ Clinical characteristics } \\
\hline Age, median (IQR), years & $30(30-32)$ \\
\hline Gestational age on admission, median (IQR), weeks & $38^{+5}\left(37^{+6}-39^{+4}\right)$ \\
\hline Epidemiological history & 19(82.6) \\
\hline Onset of symptom to delivery, median (IQR), days & $4(0-15.5)$ \\
\hline Complications & 18(78.3) \\
\hline Antenatal infection symptoms & $13(56.5)$ \\
\hline Fever & $10(43.5)$ \\
\hline Cough & $6(26.1)$ \\
\hline \multicolumn{2}{|l|}{ Diagnosis of suspected cases } \\
\hline SARS-CoV-2 nucleic acid positive & $10(43.5)$ \\
\hline SARS-CoV-2-IgM/IgG positive & $13(56.5)$ \\
\hline \multicolumn{2}{|l|}{ Laboratory Findings } \\
\hline Low or normal leukocytes $(\varangle 9.5 \times 10 \rrbracket / L)$ & $14(60.9)$ \\
\hline Neutrophilia $(\llbracket 6.3 \times 10 \rrbracket / L)$ & $15(65.2)$ \\
\hline Lymphopenia $(₫ 1.1 \times 10 \bigotimes / L 1)$ & $5(21.7)$ \\
\hline Elevated C-reactive protein $(₫ 10$ mg/L) & $5 / 22(22.7)$ \\
\hline Elevated interleukin $6(\llbracket 0-7 \mathrm{pg} / \mathrm{ml})$ & $9 / 21(42.9)$ \\
\hline 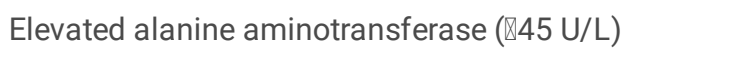 & $2(8.7)$ \\
\hline Elevated aspartate aminotransferase $(\varangle 35 \mathrm{U} / \mathrm{L})$ & $3(13.0)$ \\
\hline Elevated creatine kinase-MB $(\llbracket 0-25$ ng/ml) & $2 / 22(9.1)$ \\
\hline Elevated lactate dehydrogenase $(\varangle 243 \mathrm{U} / \mathrm{L})$ & $4 / 22(18.2)$ \\
\hline Influenza infection & 2/13(15.4) \\
\hline \multicolumn{2}{|l|}{ Chest Computed Tomographic results } \\
\hline Unilateral pneumonia & $7(30.4)$ \\
\hline Bilateral pneumonia & $16(69.6)$ \\
\hline \multicolumn{2}{|l|}{ Method of delivery } \\
\hline Caesarean section & $22(95.7)$ \\
\hline Vaginal delivery & $1(4.3)$ \\
\hline \multicolumn{2}{|l|}{ Clinical outcomes } \\
\hline Clinical cure & $23(100)$ \\
\hline Length of hospital stay, median (IQR), days & $5(4-7)$ \\
\hline
\end{tabular}

Table 2: Baseline Characteristics $\square$ Clinical Characteristics and Treatment of the Newborns 


\begin{tabular}{|c|c|}
\hline & Newboms $(\mathrm{N}=25)$ \\
\hline \multicolumn{2}{|l|}{ Clinical characteristics } \\
\hline Gestational age at delivery, median (IQR), weeks & $38^{+6}\left(37^{+6}-39^{+4}\right)$ \\
\hline \multicolumn{2}{|l|}{ Sex } \\
\hline Female & 13(52) \\
\hline Male & 12(48) \\
\hline Premature delivery & $3(12)$ \\
\hline \multicolumn{2}{|l|}{ Character of amniotic fluid } \\
\hline Clear & $15(60)$ \\
\hline Bloody amniotic fluid & $2(8)$ \\
\hline Stained amniotic fluid $\left(\mathrm{I}^{\circ}-\mathrm{II} \mathrm{I}^{\circ}\right)$ & $8(32)$ \\
\hline Birthweight, median (IQR), g & $2920(2735-3150)$ \\
\hline Neonatal asphyxia & $1(4)$ \\
\hline Fever & 0 \\
\hline \multicolumn{2}{|l|}{ Laboratory Findings } \\
\hline SARS-CoV-2 nucleic acid positive at birth & 0 \\
\hline SARS-CoV-2 nucleic acid suspected positive after birth & $3 / 24(12.5)$ \\
\hline Chest X-ray evidence of pneumonia & $0 / 24$ \\
\hline \multicolumn{2}{|l|}{ Clinical outcomes } \\
\hline Clinical cure & $23 / 24(95.8)$ \\
\hline Length of hospital stay, median (IQR), days & $8(7-9)$ \\
\hline
\end{tabular}

\section{Figures}




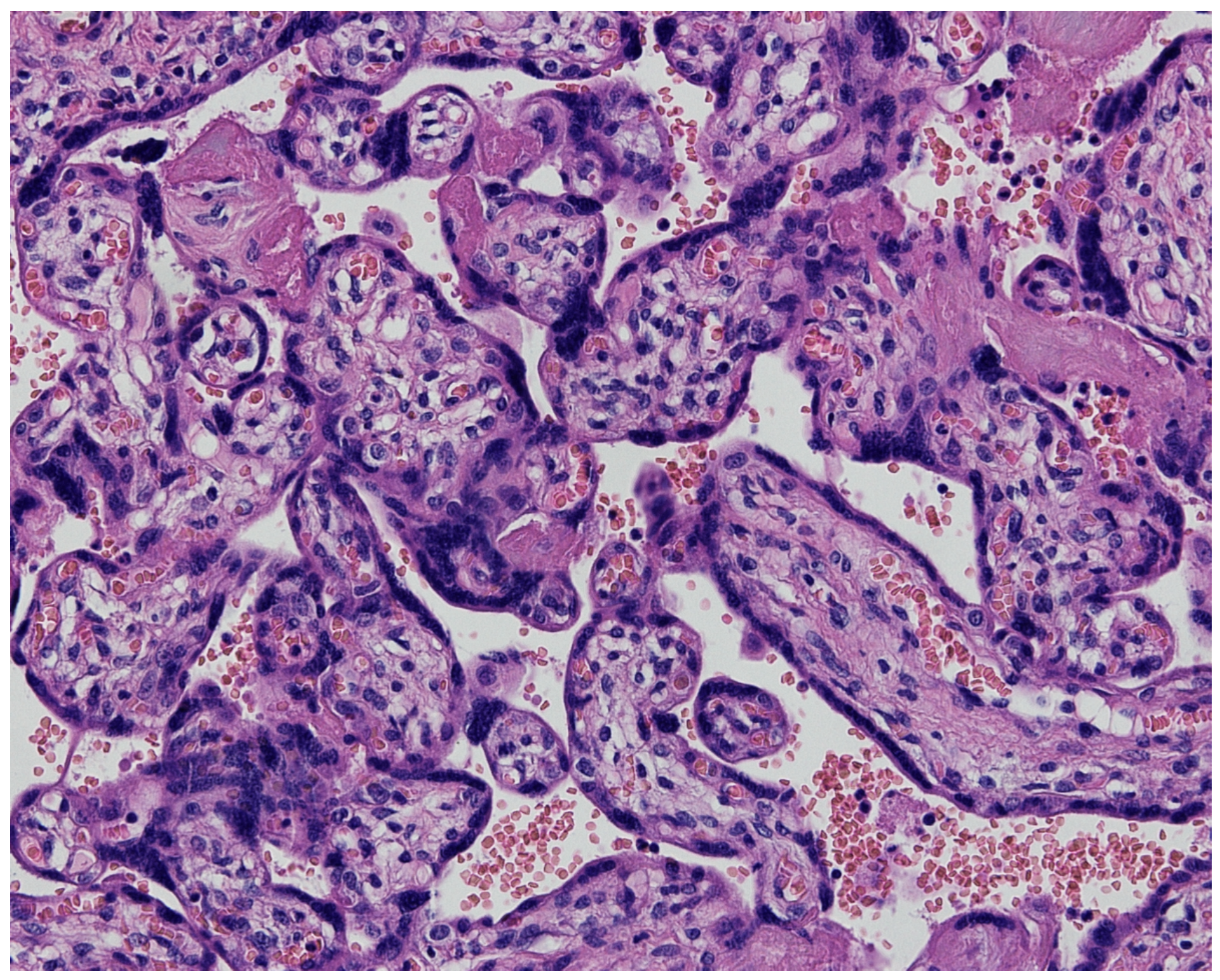

Figure 1

Pathological results of placenta in Patients Infected With SARS-CoV-2(×200). 


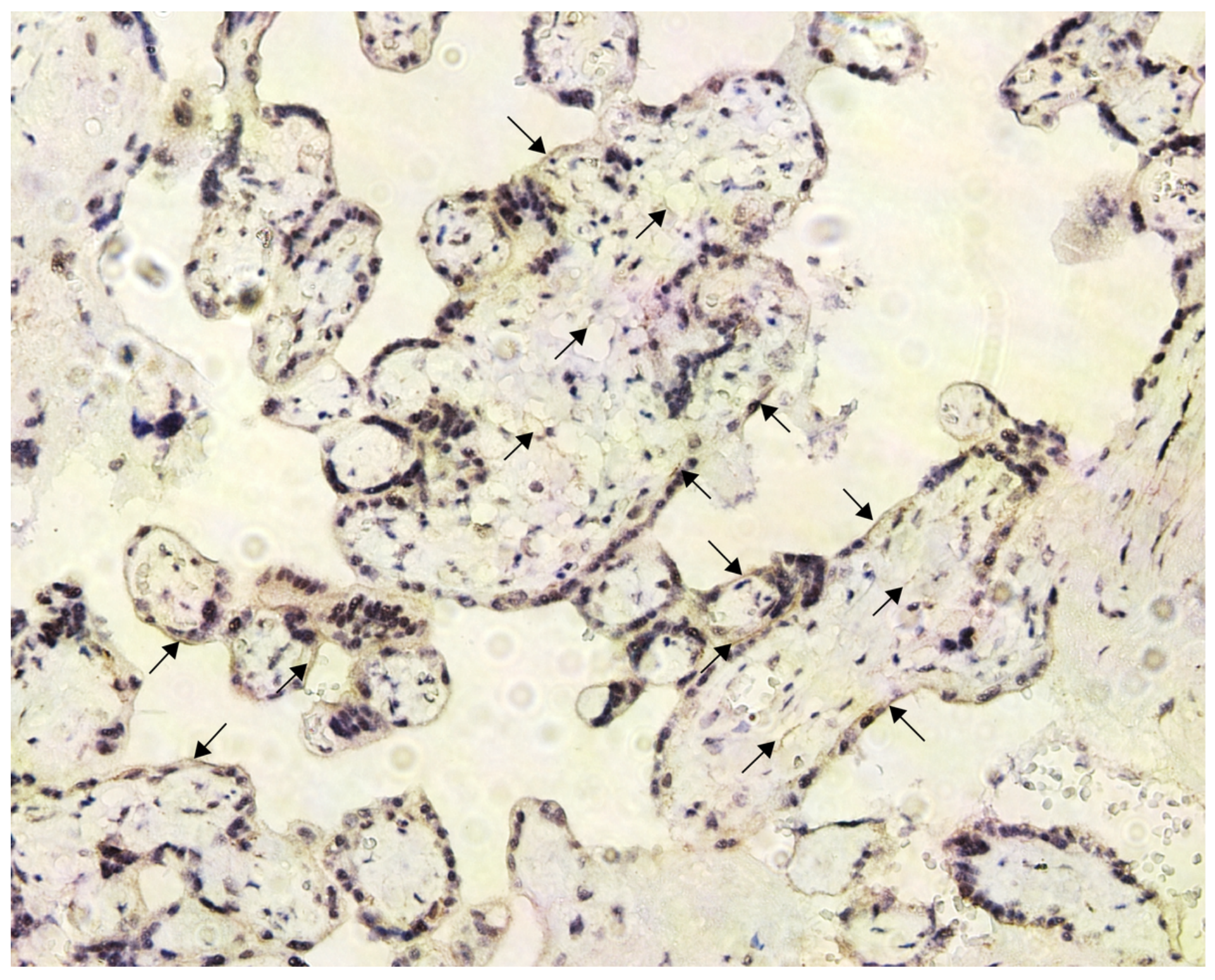

Figure 2

Immunohistochemistry of ACE2 (arrows) on placenta in Patients Infected With SARS-CoV-2(×200). 